\title{
Lactoferrin and secretory IgA levels in totally edentulous patients with two-layer complete dentures
}

\section{Mariana Yankova ${ }^{1}$, Vesela Lozanova ${ }^{2}$, Anelia Vasileva ${ }^{2}$, Bozhidar Yordanov ${ }^{3}$, Valentin Lozanov ${ }^{4}$, Ivan Mitov ${ }^{5}$}

1. Assistant Professor, Department of Prosthetic Dental Medicine, Faculty of Dental Medicine, Medical University - Sofia, Bulgaria

2. Assistant Professor, Department of Medical Chemistry and Biochemistry, Faculty of Medicine, Medical University - Sofia, Bulgaria

3. Professor, Head of Department of Prosthetic Dental Medicine, Faculty of Dental Medicine, Medical University - Sofia, Bulgaria

4. Associate Professor, Department of Medical Chemistry and Biochemistry, Faculty of Medicine, Medical University - Sofia, Bulgaria

5. Professor, Head of Department of Medical Microbiology, Faculty of Medicine, Medical University - Sofia, Bulgaria

\begin{abstract}
Introduction: The excessive bone resorption required a treatment by lined with elastic material complete dentures. Their porous structure is a prerequisite for the development of bacterial and fungal colonization. The salivary lactoferrin (LF) and secretoty immunoglobulin A (s/gA) is important for antibacterial effect of saliva and for maintaining of immune status of oral cavity.
\end{abstract}


The purpose of the present in vivo study was to establish the immunomodulatory properties of LF and slgA in totally edentulous patients, treated with complete dentures, lined with silicone-based elastic materials.

Materials and methods: The 43 totally edentulous patients, at the age of 48 - 90 years, were distributed in three experimental groups: $C,(n=15)$ treated with conventional dentures, made only of rigid acrylic resin; $A,(n=15)$ and $B,(n=13)$ treated with dentures lined with heat-polymerized [Molloplast $B$ (Detax, Germany)] and auto-polymerized [Megabase (Dreve, Germany)] silicone-based elastic materials, respectively. For quantitative determination of $L F$ and slgA concentration in salvia, before and 3 months after prosthetic treatment, were used commercial kits: Human Lactoferrin ELISA kit and Human IgA ELISA kit, MyBioSource, Inc, San Diego, CA, USA.

Results: After prosthetic treatment we established increased salivary level of $L F$ from $0.266 \pm 0.006$ to $0.282 \pm 0.008 \mu \mathrm{g} / \mathrm{ml}, p<0.05$ in group $B$ patients and decrease of slgA in group $C$ patients from 165.81 \pm 5.50 to $146.91 \pm 4.13 \mu \mathrm{g} / \mathrm{ml}, p<0.01$. The $L F$ and slgA concentrations did not change in patients of group $A$.

Conclusion: The immunomodulatory role of slgA or LF has been demonstrated more pronounced in patients treated with acrylic resin complete dentures and those lined with auto-polymerized siliconebased elastic material.

Keywords: Lactoferrin, slgA, saliva, soft denture liner, two-layer complete dentures, imunomodulatoty function

\section{Introduction}

Advanced bone resorption, presence of painful neurogenic spots, thin non-resilient mucosa, established in totally edentulous patients, require the use of more specific methods of prosthetic treatment, namely the making of two-layer complete dentures (acrylic resin complete dentures, lined with elastic material). These denture constructions distribute more evenly the chewing pressure and thus lessen the trauma on the mucosa $(1,2)$. Their main disadvantage is that their porous structure is a prerequisite to bacterial and fungal colonization in the oral cavity $(3,4,5)$, while the qualities and the quantities of composition of the saliva is important factor of this $(6,7)$.

The antibacterial activity of saliva is determined mainly by histidine-rich peptides, peroxidase system activity, lysozyme, lactoferrin, secretory IgA, etc. (8). They reduce the growth of microorganisms and fungi, affect the bacterial glycolic activity and by aggregation and elimination of some microorganisms they participate in the maintenance of mouth cavity homeostasis $(9,10,11)$.

Lactoferrin (LF) is a non-enzyme glycoprotein of the transferrin family, with high affinity towards iron, and is found in the exocrine glands' secretions and in specific granules in neutrophils (12). This explains the established lower LF levels in totally edentulous patients (13). Lactoferrin has fungicidal, antiviral and antiinflammatory effects. It is considered to be a part of the innate immune response and to have immune modulating function $(8,14,15)$. Many authors report change in Lactoferrin values in different local diseases $(16,17,18,19,20,21)$. 
Main component of the mucosal immune system is the secretory $\lg A(\lg A)$, which is used for evaluating the immune status of the oral cavity $(22,23,24)$. Rashkova et al., (25) observe increase in the slgA values in children carrying removable orthodontic appliances. The authors concluded that the orthodontic appliances are a local immunogenic factor, which is a stronger stimulus for the oral immunity than systemic factors as diabetes and asthma. Major part of the salivary IgA is secretory and a very small part comes from creviculare fluid, i.e. is serum originated. According to the study by Karova et al., (7) the concentration of salivary immunoglobulins does not change during aging, but on the other hand available data show a reduced response to antigenic stimulus after 60-65 years of age, which may be a condition for developing an oral candidiasis in these patients.

A number of authors found higher levels of IgA in totally edentulous patients $(26,27)$. Eliasson et al. has established correlation between gender and salivary concentration of slgA, as lower values of IgA are reported in women (26).

The purpose of the present in vivo study was to establish the immunomodulatory properties of LF and slgA in totally edentulous patients, treated with complete dentures, lined with silicone-based elastic materials.

\section{Materials and methods}

Patients: 43 totally edentulous patients at the age of 48 to 90 (average age of $68.4 \pm 9.94$ ), 12 men and 31 women, were examined. All patients signed informed consent form for participation in the present research. The scientific research was approved by the Research Ethics Commission "KENIMUS" (Statement № 21/2016). The patients were distributed in three groups according to the material their dentures were made of. First, control group (group C) - patients treated with conventional complete dentures (made only of rigid acrylic resin) in both upper and lower jaw, $(n=15)$. Second group (group A) patients, treated with complete dentures - conventional denture on the upper jaw, and on the lower jaw denture, lined with heat-polymerized silicone-based elastic material [Molloplast B (Detax, Germany)], $(n=15)$. Third group (group B) - patients, treated with complete dentures - conventional denture on the upper jaw, and on the lower jaw - denture, lined with auto-polymerized silicone-based elastic material [Megabase (Dreve, Germany)], $(n=13)$.

Saliva collection: A sample of unstimulated whole saliva, for biochemical analysis, was collected from all patients before denture delivery and on the third month after prosthetic treatment. The samples were taken following the A.Vissink method (7): always in the morning between 09:00 and 12:00 o'clock, patients were instructed in advance not to eat, drink or smoked for one hour before collection appointments. Before the saliva collection, the patients washed their mouths with deionized water. The saliva was collected by the method of spitting (the patients gathered saliva at the bottom of their oral cavity and spitted it at intervals of $60 \mathrm{~s}$ in a $15 \mathrm{ml}$ plastic container). The needed quantity of about $2 \mathrm{ml}$ was placed in a crio-test tube. The samples were immediately frozen in liquid nitrogen and were kept in $-70^{\circ} \mathrm{C}$ until the analysis performance. 
Biochemical analysis: For the quantitative determination of Lactoferrin and secretory Immunoglobulin A (sIgA) levels in saliva were used commercial ELISA kits (Human Lactoferrin ELISA kit and Human IgA ELISA kit, both from MyBioSource,Inc, San Diego, CA, USA) and the analyses were performed following the manufacturer's protocols. Using the results for the calibration standards provided in the kits were constructed standard quadratic curves for Lactoferrin and for $\operatorname{sig} A\left(R^{2}=0.9702\right.$ and $R^{2}=0.9968$, respectively) and the amounts of Lactoferrin and slgA in the biological samples were calculated based on the respective equations. The amount of Lactoferrin and slgA in the analyzed biological samples is expressed as $\mu \mathrm{g} / \mathrm{ml}$ and represents the mean value of two independent measurements. All data are expressed as mean \pm SEM.

For the statistical analysis we used a computer configuration SPSS version 19. For analysis of the results and for comparison of the examined parameters we used Student t-test with 95\% confidence interval $(p<$ 0.05).

\section{Results}

In the first step of our study we investigated whether the salivary concentrations of LF and slgA in patients, included in the current study, before prosthetic treatment, are sex or age dependant. Before prosthetic treatment we did not establish sex differences of LF and slgA salivary levels in investigated patients. The LF and sIgA concentrations in man were: $0.268 \pm 0.011$ and $165.64 \pm 6.67$; in woman were: $0.271 \pm 0.005$ and $163.95 \pm 6.67 \mu \mathrm{g} / \mathrm{ml}$, respectively. In studying the age dependency of salivary levels of LF and slgA before prosthetic treatment, we established lack of differences between four age-distributed patients group: $48-58,58-68,68-78$ and over 78 years of age, respectively (Table 1 ).

Table 1. Concentration of Lactoferrin (LF) and secretory immunoglobulin A (slgA) in saliva ( $\mu \mathrm{g} / \mathrm{ml})$ before prosthetic treatment in age-distributed patients group.

\begin{tabular}{lcc}
\hline \hline Age & LF & slgA \\
\hline 48-58 years $(\mathbf{n}=\mathbf{7})$ & $0.269 \pm 0.015$ & $156.60 \pm 6.73$ \\
$\mathbf{5 8 - 6 8}$ years $(\mathbf{n}=\mathbf{1 4})$ & $0.264 \pm 0.006$ & $161.78 \pm 6.76$ \\
$\mathbf{6 8 - 7 8}$ years $(\mathbf{n = 1 3})$ & $0.268 \pm 0.008$ & $166.98 \pm 5.28$ \\
over 78 years of age $(\mathbf{n}=\mathbf{9})$ & $0.263 \pm 0.007$ & $156.08 \pm 5.39$ \\
\hline \hline
\end{tabular}

The second step of our study investigated immunomodulatory properties of LF and slgA in totally edentulous patients, treated with complete dentures made only of rigid acrylic resin in comparison to patients, treated with dentures, lined with different silicone-based elastic materials: auto-polymerized and heat-polymerized silicone-based elastic materials. Our results showed lack of differences in salivary levels in LF as well as in sIgA before prosthetic treatment between main C, A and B investigated experimental groups (Table 2). Three months after prosthetic treatment we established decrease of salivary 
concentration of slgA in patients treated with complete denture made only of rigid acrylic resin (Group C) from $165.81 \pm 5.50$ to $146.91 \pm 4.13 \mu \mathrm{g} / \mathrm{ml}, \mathrm{p}<0.01$. In patients treated with complete dentures, lined with auto-polymerized silicone-based elastic material (Group B) we established increase of salivary levels of LF from $0.266 \pm 0.006$ to $0.282 \pm 0.008 \mu \mathrm{g} / \mathrm{ml}, p<0.05$. In patients, treated with dentures, lined with heatpolymerized silicone-based elastic material (group A), we did not establish differences in LF as well as in slgA salivary levels before and after prosthetic treatment.

Table 2. Concentration of Lactoferrin (LF) and secretory immunoglobulin A (slgA) in saliva ( $\mu \mathrm{g} / \mathrm{ml})$ before and after prosthetic treatment in experimental groups $C, A$ and $B$

\begin{tabular}{lcccc}
\hline \hline & \multicolumn{2}{c}{ LF } & \multicolumn{2}{c}{ slgA } \\
\cline { 2 - 5 } & $\begin{array}{c}\text { Before } \\
\text { prosthetic } \\
\text { treatment }\end{array}$ & $\begin{array}{c}\text { After } \\
\text { prosthetic } \\
\text { treatment }\end{array}$ & $\begin{array}{c}\text { Before } \\
\text { prosthetic } \\
\text { treatment }\end{array}$ & $\begin{array}{c}\text { After } \\
\text { prosthetic } \\
\text { treatment }\end{array}$ \\
\hline $\begin{array}{l}\text { Group C } \\
(\mathbf{n = 1 5 )}\end{array}$ & $0.272 \pm 0.012$ & $0.275 \pm 0.006$ & $165.81 \pm 5.50$ & $146.91 \pm 4.13^{\star *}$ \\
\hline $\begin{array}{l}\text { Group A } \\
(\mathbf{n = 1 5 )}\end{array}$ & $0.273 \pm 0.007$ & $0.265 \pm 0.003$ & $159.75 \pm 4.36$ & $162.21 \pm 3.14$ \\
\hline $\begin{array}{l}\text { Group B } \\
(\mathbf{n}=13)\end{array}$ & $0.266 \pm 0.006$ & $0.282 \pm 0.008^{*}$ & $158.00 \pm 5.69$ & $155.47 \pm 3.26$ \\
\hline \hline
\end{tabular}

* $p<0.05$ - statistically significant differences in salivary LF concentration before vs. after prosthetic treatment

** $p<0.01$ - statistically significant differences in salivary slgA concentration before vs. after prosthetic treatment

Group C - patients treated with conventional complete dentures (made only of rigid acrylic resin) in both upper and lower jaw $(n=15)$;

Group A - patients treated with complete dentures - conventional denture on the upper jaw, and on the lower jaw - denture lined with heat-polymerized silicone-based elastic material [Molloplast B (Detax, Germany)], ( $n=15)$;

Group B - patients treated with complete dentures - conventional denture on the upper jaw, and on the lower jaw - denture lined with auto-polymerized silicone-based elastic material [Megabase (Dreve, Germany)], ( $n=13)$; 
Figure 1. Concentration of Lactoferrin (LF) and secretory immunoglobulin A (slgA) in saliva ( $\mu g / \mathrm{ml})$ before and after prosthetic treatment in man and woman distributed in group $C, A$ and $B$.
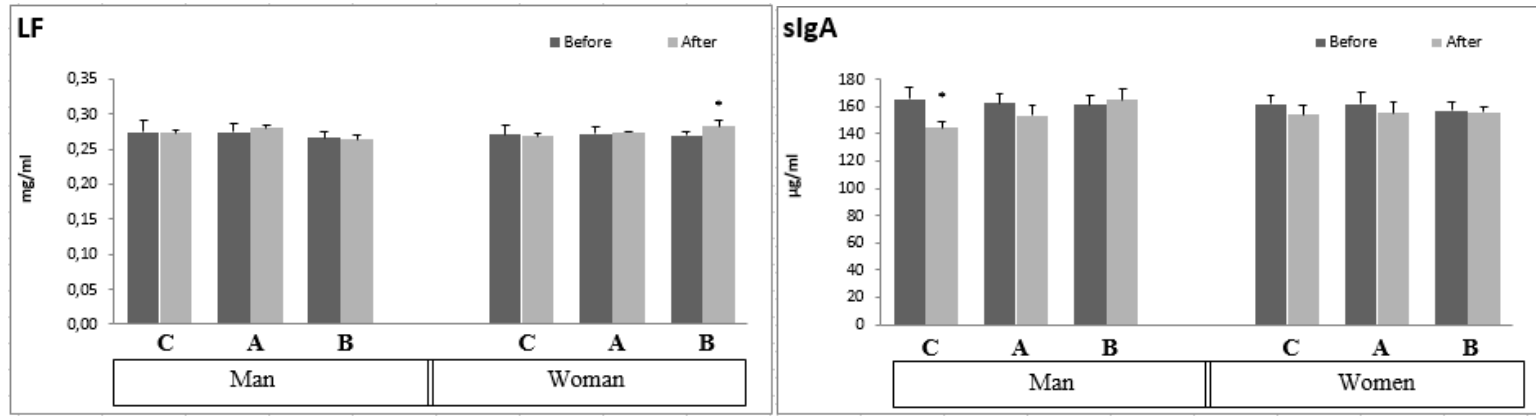

${ }^{*} p<0.05$ - statistically significant differences in salivary LF or slgA concentrations before vs. after prosthetic treatment

Group C: Man, $(n=5)$; Woman, $(n=10)$ - patients treated with conventional complete dentures (made only of heat-polymerized rigid acrylic resin) in both upper and lower jaw;

Group A: Man, $(n=5)$; Woman, $(n=10)$ - patients treated with complete dentures - conventional denture on the upper jaw, and on the lower jaw - denture lined with heat-polymerized siliconebased elastic material [Molloplast B (Detax, Germany)];

Group B : Man, $(n=5)$; Woman, $(n=8)$ - patients treated with complete dentures - conventional denture on the upper jaw, and on the lower jaw - denture lined with auto-polymerized siliconebased elastic material [Megabase (Dreve, Germany)];

The results obtained in our study do not indicate an age-dependent response to investigated different materials, used in denture.

In addition, we examined whether the use of partial or complete dentures over the past 5 years, led to changes of immunomodulation action of LF or slgA in saliva in investigated patients. We have established increased concentration of LF in patients treated with partial or complete dentures $(P+, n=33): 0.271 \pm$ $0.004 \mu \mathrm{g} / \mathrm{ml}$ compared to patients who have never used either partial or complete dentures $(P-, n=10)$ : $0.245 \pm 0.006 \mu \mathrm{g} / \mathrm{ml}, \mathrm{p}<0.05$, (Fig. 2). In P+ group patient's salivary LF concentration did not change 3 monads after prosthetic treatment. Interestingly, in $\mathrm{P}$ - group patients, 3 monads after prosthetic treatment LF concentration in saliva increased statistically significant to $0.270 \pm 0.003 \mu \mathrm{g} / \mathrm{ml}, p<0.01$, which does not differ from the level of LF in patients in a group $\mathrm{P}+$.

The salivary slgA concentrations in patients of group $\mathrm{P}+$ and group $\mathrm{P}$ - did not differ: $163.75 \pm 3.67$ and $158.16 \pm 2.38 \mu \mathrm{g} / \mathrm{ml}$, respectively. However, 3 monads after prosthetic treatment salivary slgA level in group P-patients decreased to $145.16 \pm 4.42 \mu \mathrm{g} / \mathrm{ml}$, p<0.05, (Fig. 2). 
Figure 2. Concentration of Lactoferrin (LF) and secretory immunoglobulin A (slgA) in saliva ( $\mu g / \mathrm{ml})$ before and after prosthetic treatment of patients used partial or complete dentures over the past 5 years $(P+, n=33)$ and patients who have never used either partial or complete dentures $(P-, n=10)$.
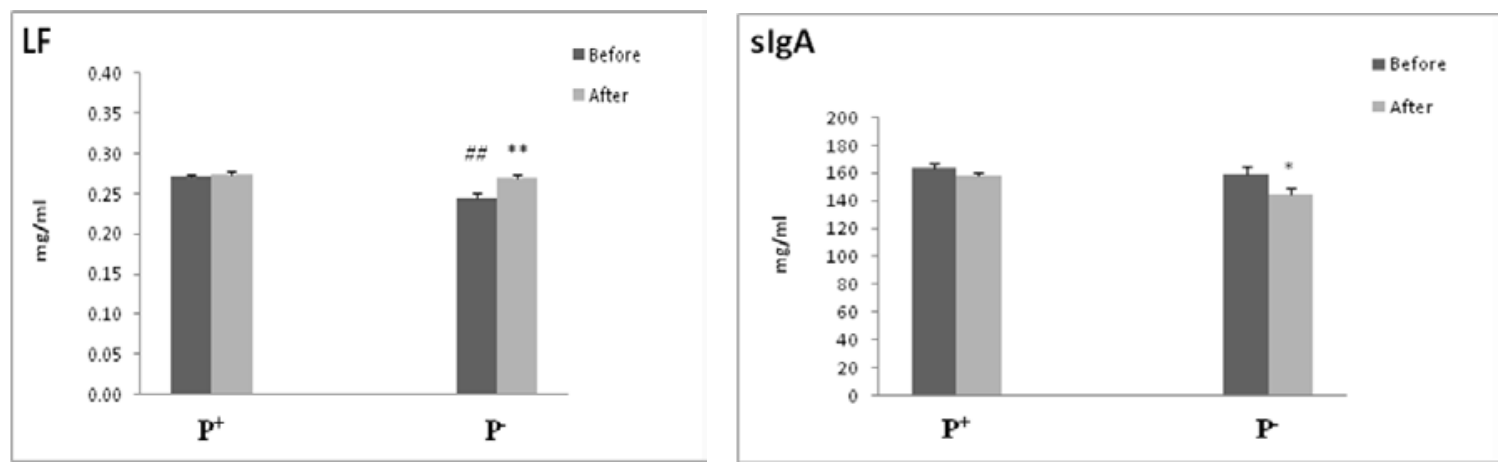

\#\# $\mathrm{p}<0.01$ - statistically significant differences in salivary LF concentration between P+ vs. P- patients group

** $p<0.01$ - statistically significant differences in salivary LF concentration before vs. after prosthetic treatment in P-patients group

${ }^{*} p<0.05$ - statistically significant differences in salivary slgA concentration before vs. after prosthetic treatment in P- patients group

We have established that prosthetic treatment led to increase of immunomodulatory role of LF for extended period of time and to decrease of immunomodulatory action of slgA for short term interval. These results demonstrated specificity in the dynamics of salivary LF and slgA regulation.

\section{Discussion}

In the curent study we investigate the salivary levels of LF and sIgA, before treatment and 3 month after treatment of totally edentulous patients with complete dentures made only of rigid acrylic resin in comparison to treatment with dentures, lined with two different types of silicone-based elastic materials: auto-polymerized and heat-polymerized silicone-based elastic materials. In the period before prosthetic treatment in investigated totally edentulous patients we established lower levels of salivary Lactoferrine, in comparison to those, reported in the scientific literature. Närgi at all. (13) report lower values in edentulous patients - mean $13.2 \pm 8.0 \mathrm{mg} / \mathrm{l}$. Other authors established values of LF from $8.96 \mathrm{mg} / \mathrm{l}$ to $10.54 \mathrm{mg} / \mathrm{l}(16$, $17,18,19)$. The lowest values $(1.5 \mu \mathrm{g} / \mathrm{ml}$ in children) were established by Harrison \& Bowen $(21)$. As the current study of salivary levels of LF and slgA in totally edentulous adult patients is performed for the first time in our country, we did not have the opportunity to compare our results with other similar to them. We assume that low salivary LF concentration $(0.268 \pm 0.004 \mu \mathrm{g} / \mathrm{ml})$, established in our study are probably determined by demographic factors. The values of salivary slgA commented in the science literature vary in the diapason from $110.0 \mu \mathrm{g} / \mathrm{ml}$ in totally edentulous (27) to $208.8 \mu \mathrm{g} / \mathrm{ml}$ in children with orthodontic 
appliances (25). The established in current study salivary levels of slgA (162.69 \pm 3.08$)$ in totally edentulous adult patients is compatible with these reported in other investigation.

We did not establish sex as well as age-specific differences of LF and slgA salivary levels in investigated patients before prosthetic treatment. The available results, obtained from the investigation of salivary levels of slgA are controversial. It has been reported age-depended reduced or unchanged response of slgA in saliva to different antigenic stimulus (7). Other authors established lower values of salivary slgA in women (26). The LF and slgA have an important immune modulating function. It is a possible difference of available data for salivary levels LF and slgA is the result of presence local or chronic diseases, changed immune status of the oral cavity in investigated patients. It has been established that diabetes and asthma, as well as removable orthodontic appliances, affected oral immunity responses $(7,16,17,18,19$, $20,21,22,23,25)$.

The results obtained in our study demonstrate that prosthetic treatment with complete dentures, lined with auto-polymerized silicone-based elastic material (group B) increased salivary concentration of LF while prosthetic treatment with dentures, lined with heat-polymerized silicone-based elastic materials (group A) did not affect LF levels. On the other hand we established that treatment with conventional denture made only of rigid acrylic resin (group $C$ ) decreased salivary slgA concentration. These results demonstrated specific response of oral immune function mediating by LF and slgA to soft liner denture and to conventional denture respectively. The prosthetic treatment with conventional denture made of rigid acrylic resin affected main component of the mucosal immune system - salivary slgA. This may be considered as a response to the action of a local immunogenic factor.

The prosthetic treatment with complete dentures lined with auto-polymerized silicone-based elastic material led to increase salivary concentration of LF. The fungicidal, antiviral and anti-inflammatory effects of LF were established $(8,14,15)$. It is possible that the porous structure of silicone-based elastic materials causes bacterial or fungal colonization in the oral cavity $(3,4,5)$, which in turn leads to activation of LF. On the other hand we established lack of changes in the immune response mediated by LF and slgA after prosthetic treatment with different type of dentures, lined also with silicone-based elastic material but heat-polymerized. It is possible that this type of dentures may cause activation of other factors involved in maintaining oral immune status. It is not excluded that the effects on LF or slgA may occur later in the period after prosthetic treatment.

In our study we established sex specific response to materials used in complete dentures. The treatment with denture made only of rigid acrylic resin provoked more pronounced inhibitor effect on immunomodulation action of slgA in man. In women, treated with dentures lined with auto-polymerized silicone-based elastic material, the increased immunomodulation role of LF is underlined. We did not establish age specific response to prosthetic treatment with dentures made of investigated different materials.

\section{Conclusion}

The treatment with complete dentures lined with heat-polymerized silicone-based elastic materials did not change immunomodulatory properties of LF and slgA in saliva 3 months after prosthetic treatment. In contrast, the prosthetic treatment with complete dentures, lined with auto-polymerized silicone-based elastic material as well as prosthetic treatment with conventional complete dentures affected the important 
investigated components maintaining the immune status of oral cavity - LF and slgA. The prosthetic treatment with dentures, lined with auto-polymerized silicone-based elastic material or prosthetic treatment with conventional complete dentures lead to sex specific response of LF and slgA in saliva.

\section{Acknowledgement}

This study is financially supported through Research Project № 5085/2016 from the Medical University Sofia, Bulgaria.

\section{References}

1. Grant A.A., Heath J.R., McCord J.F.: Complete prosthodontics - problems, diagnosis and management. Wolfe, 1994:123-125.

2. Hayakawa I.: Principles and Practices of Complete Dentures. Creating the mental image of a denture. Quintessence Publishing. 1999: 233-248.

3. Mutluay M.M. et al.: A Prospective Study on the Clinical Performance of Polysiloxane Soft Liners: One-year Results. Dental Materials Journal. 2008, 27(3):440-447.

4. Marxkors R.(Hrsd.): Lehrbuch der zahnärztlichen Prothetik.5. Auflage. Deutscher Zahnärzte Verlag Köln, 2010:359.

5. Valentini F., Luz M.S., Boscato N., Pereira-Cenci T. : Biofilm formation on denture liners in a randomised controlled in situ trial. J Dent. 2013, 41(5):420-427.

6. Boscato $\mathrm{N}$ et al.: Biofilm formation of Candida albicans on the surface of a soft denture-lining material. Gerodontology. 2009, 26(3):210-213.

7. Karova E.: Saliva - factor for maintaining of the oral homeostasis. 2013: 19-40, 41-46, $51-56$ (in Bulgarien).

8. De Almeida PDV. et al.: Saliva Composition and Function: A Comprehensive Review. J Contemp Dent Pract. 2008, 9(3):072-080.

9. Krasteva-Panova A.Z.: Whole unstimulated saliva as a diagnostic medium for immuneinflammatory diseases and oral neoplasms. Dissertation, Sofia, 2009, 13-15 (in Bulgarien).

10. Mandel ID.: The functions of saliva. J Dent Res. 1987, 66(Spec Iss 2):623-627.

11. Velliyagounder K. : Role of Human Lactoferrin in Oral Diseases. Oral Hyg Heallth. 2014, 2(1):1000e108.

12. Masson PL, Heremans JF, Schonne E.: Lactoferrin, an iron-binding protein in neutrophilic leukocytes. J Exp Med. 1969, 130(3):643-58.

13. Närhi TO, Tenovuo J, Ainamo A, Vilja P.: Antimicrobial factors, sialic acid, and protein concentration in whole saliva of the elderly. Scand J Dent Res. 1994 Apr;102(2):120-125.

14. Nikolova M, Nikolov P, Baleva M: Lactoferrin in Clinical Practice. Medical examination, 2014, 50(3):65-68 (in Bulgarien).

15. Edgerton M., Koshlukova SE.: Salivary Histatin 5 and its similarities to the other antimicrobial proteins in human saliva. Adv Dent Res. 2000, 14:16-21.

16. Fine $\mathrm{DH}$, Furgang D, Beydouin F. Lactoferrin iron levels are reduced in saliva of patients with localized aggressive periodontitis. J Periodontol. 2002, 73(6):624-630.

17. Berlutti F, Pilloni A, Pietropaoli M, Polimeni A, Valenti P. Lactoferrin and oral diseases: current status and perspective in periodontitis. Annali di Stomatologia. 2011; 2(3-4):10-18.

18. Jentsch $\mathrm{H}$, Sievert $\mathrm{Y}$, Göcke R.: Lactoferrin and other markers from gingival crevicular fluid and saliva before and after periodontal treatment. J Clin Periodontol. 2004; 31(7):511-514. 
19. Groenink J, Walgreen-Weterings E, Nazmi K, Bolscher JG, Veerman EC, van Winkelhoff AJ, Nieuw Amerongen AV.: Salivary lactoferrin and low-Mr mucin MG2 in Actinobacillus actinomycetemcomitans-associated periodontitis. J Clin Periodontol. 1999; 26(5):269-275.

20. Moslemi M, Sattari M, Kooshki F, et al. Relationship of Salivary Lactoferrin and Lysozyme Concentrations with Early Childhood Caries. Journal of Dental Research, Dental Clinics, Dental Prospects. 2015; 9(2):109-114.

21. Harrison R, Bowen $\mathrm{WH} .:$ Flow rate and organic constituents of whole saliva in insulin-dependent diabetic children and adolescents. Pediatr Dent. 1987, 9(4):287-291.

22. Krasteva A, Perenovska P, Ivanova A, Altankova I, Bocheva T, Kisselova A.: Alteration in Salivary Components of Children with Allergic Asthma. Biotechnology\& Biotechnological Equipment , 2010, 24(2):1866-1869.

23. Marcotte H., Lavoie MC.: Oral microbial ecology and the role of salivary immunoglobulin A. Microbiol Mol Biol Rev. 1998, 62(1):71-109.

24. Saluja R, Kale A, Hallikerimath S. : Determination of levels of salivary IgA subclasses in patients with minor recurrent aphthous ulcer. J Oral Maxillofac Pathol. 2012, 16(1):49-53.

25. Rashkova $M$ et al.: Secretory immunoglogulin $A(S-\lg A)$ in the Saliva of children withType 1 Diabetes, Asthma, Systemic Health and Systemic Health but Wearing Removable Orthodontic Appliances. OHDMBSC. 2009, VIII(2), 16-24.

26. Eliasson L, Birkhed D, Osterberg T, Carlén A.: Minor salivary gland secretion rates and immunoglobulin A in adults and the elderly. Eur J Oral Sci. 2006; 114(6):494-499.

27. Pajukoski H, Meurman JH, Snellman-Gröhn S, Keinänen S, Sulkava R.: Salivary flow and composition in elderly patients referred to an acute care geriatric ward. Oral Surg Oral Med Oral Pathol Oral Radiol Endod. 1997; 84(3):265-271.

\title{
Corresponding author:
}

\author{
Mariana Yankova, \\ Department of Prosthetic Dental Medicine, \\ Faculty of Dental Medicine, \\ Medical University, \\ 1 G. Sofiiski Blvd., \\ Sofia, Bulgaria \\ email: m.jankova@abv.bg
}

